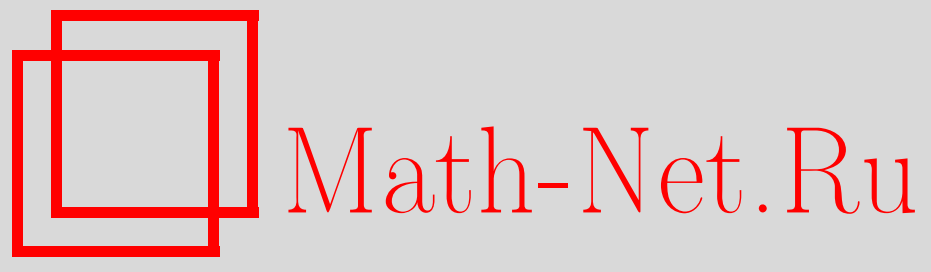

Г. А. Калябин, Точные константы в одномерных неравенствах типа Пуанкаре, Матем. заметки, 2011, том 90, выпуск 4, 634-636

DOI: https://doi.org/10.4213/mzm9063

Использование Общероссийского математического портала Math-Net.Ru подразумевает, что вы прочитали и согласны с пользовательским соглашением http://www . mathnet.ru/rus/agreement

Параметры загрузки:

IP : 54.237 .206 .68

26 апреля 2023 г., 02:30:07

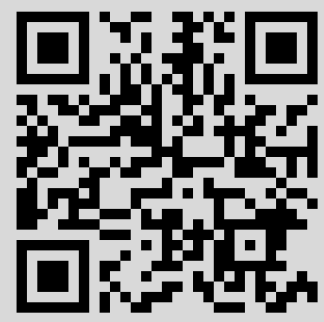




\section{Точные константы в одномерных неравенствах типа Пуанкаре}

\section{Г. А. Калябин}

1. Обозначения и формулировка результата. Пусть $W_{2}^{1}(-1,1)$ - пространство Соболева всех вещественнозначных функций $f(t)$, абсолютно непрерывных на отрезке $[-1,1]$ и таких, что $f^{\prime} \in L_{2}(-1,1)$, снабженное полунормой

$$
\|f\|_{W_{2}^{1}(-1,1)}:=\left\|f^{\prime}\right\|_{L_{2}(-1,1)}:=\left(\int_{-1}^{1}\left(f^{\prime}(t)\right)^{2} d t\right)^{1 / 2} .
$$

Для $m \in \mathbb{N}$ обозначим через $W_{2, m}^{1}$ подпространство в $W_{2}^{1}(-1,1)$, состоящее из всех функций $f$, ортогональных пространству алгебраических полиномов степени $\leqslant m-1$, т.е. удовлетворящих $m$ интегральным условиям

$$
\int_{-1}^{1} t^{k} f(t) d t=0, \quad k \in\{0,1, \ldots, m-1\}
$$

На этих подпространствах величина (1) является нормой.

Наша цель - для произвольного $x \in[-1,1]$ найти наименьшие возможные константы $B_{m}(x)$ в неравенствах

$$
|f(x)| \leqslant B_{m}(x)\left\|f^{\prime}\right\|_{L_{2}(-1,1)} \quad \text { для всех } f \in W_{2, m}^{1} .
$$

Частичный ответ на данный вопрос содержится в следующем утверждении.

Теорема. Имеют место равенства
(i) $B_{m}^{2}( \pm 1)=\frac{2}{m(m+2)}$,
(ii) $B_{1}^{2}(x)=\frac{1+3 x^{2}}{6}$,
(iii) $B_{2}^{2}(0)=\frac{1}{6}$

2. Доказательство. Отметим, прежде всего, что числа $B_{m}(x)$ связаны с решением экстремальной задачи, а именно

$$
B_{m}^{-2}(x)=\min \left\{J[y]: y \in W_{2, m}^{1}, y(x)=1\right\}, \quad J[y]:=\int_{-1}^{1}\left(y^{\prime}(t)\right)^{2} d t .
$$

Кроме того, очевидно, что $B_{m}(-x)=B_{m}(x)$.

Более простым является случай (i), когда $x$ есть концевая точка отрезка.

Лемма 1. Для $x=1$ минимум функционала $J[y]$ в (5) достигается на функции

$$
\widetilde{y}_{m}(t)=\widetilde{y}_{m}(1 ; t):=\frac{(m+2) P_{m}(t)+m P_{m+1}(t)}{2(m+1)},
$$

где $P_{n}(t)$ - классические многочлены Лежандра, и при зтом

$$
J\left[\widetilde{y}_{m}\right]=\frac{m(m+2)}{2} .
$$

Работа выполнена при поддержке Российского фонда фундаментальных исследований (гранты №oo 11-01-00744a, 09-01-00093, 10-01-091331) и Немецкого исследовательского общества (грант DFG GZ SCHM 969/12-1). 
Действительно, экстремали изопериметрической задачи

$$
J[y] \rightarrow \min , \quad \int_{-1}^{1} t^{k} y(t) d t=\alpha_{k}, \quad \alpha_{k} \in \mathbb{R}, \quad k \in\{0,1, \ldots, m-1\}, \quad y(1)=1,
$$

должны удовлетворять условию трансверсальности на свободном конце $\left(y^{\prime}(-1)=0\right)$ (см., например $[1 ; 1.5 .4])$ и уравнению Эйлера $y^{\prime \prime}(t)=Q_{m-1}(t), t \in(-1,1)$, где $Q_{m-1}(t)$ - произвольный алгебраический многочлен степени $\leqslant m-1$, и, следовательно, $\widetilde{y}_{m}(t)=Q_{m+1}(t)$.

Поскольку в силу $(2)$ все числа $\alpha_{k}$ в (8) равны 0 , многочлен $Q_{m+1}(t)$ с необходимостью должен быть линейной комбинацией двух последовательных многочленов Лежандра $P_{m}(t)$ и $P_{m+1}(t)$ :

$$
\widetilde{y}_{m}(t)=c_{m, 0} P_{m}(t)+c_{m, 1} P_{m+1}(t) .
$$

Для вычисления коэффициентов $c_{m, 0}$ и $c_{m, 1}$ примем во внимание записанные ранее краевые условия $\widetilde{y}_{m}(1)=1, \widetilde{y}_{m}^{\prime}(-1)=0$ и классические равенства (см., например, $\left.[2 ; 10.10]\right)$ $P_{n}(1)=1, P_{n}^{\prime}( \pm 1)=( \pm 1)^{n-1} n(n+1) / 2$, что приводит к системе линейных уравнений

$$
c_{m, 0}(t)+c_{m, 1}=1, \quad m c_{m, 0}-(m+2) c_{m, 1}=0,
$$

решение которой легко находится:

$$
c_{m, 0}=\frac{m+2}{2(m+1)}, \quad c_{m, 1}=\frac{m}{2(m+1)},
$$

откуда непосредственно вытекает (6).

Далее, интегрируя по частям, получаем

$$
J\left[\widetilde{y}_{m}\right]=\int_{-1}^{1}\left(\widetilde{y}_{m}^{\prime}(t)\right)^{2} d t=\left.\widetilde{y}_{m}^{\prime}(t) \widetilde{y}_{m}(t)\right|_{-1} ^{1}-\int_{-1}^{1} \widetilde{y}_{m}^{\prime}(t) \widetilde{y}_{m}(t) d t=\widetilde{y}_{m}^{\prime}(1)
$$

поскольку по построению $\widetilde{y}_{m}(t)$ ортогональна $\widetilde{y}_{m}^{\prime}(t)$. Наконец,

$$
J\left[\widetilde{y}_{m}\right]=\widetilde{y}_{m}^{\prime}(1)=c_{m, 0} P_{m}^{\prime}(1)+c_{m, 1} P_{m+1}^{\prime}(1)=\frac{m(m+2)}{2} .
$$

В частности,

$$
\widetilde{y}_{1}(1 ; t)=\frac{3}{8} t^{2}+\frac{3}{4} t-\frac{1}{8}, \quad B_{1}^{2}( \pm 1)=\frac{2}{3} .
$$

3. Доказательство части (ii): $m=1,-1<x<1$. С помощью растяжений и сдвигов из (7) выводится

Лемма 2. Пусть $a<b, Y$ - множество таких функиий $y(t) \in W_{2}^{1}(a, b)$, которые равны 1 на одном из концов отрезка $[a, b], c \in \mathbb{R}$. Тогда

$$
\min _{y \in Y}\left\{\int_{a}^{b}\left(y^{\prime}(t)\right)^{2} d t: \frac{1}{b-a} \int_{a}^{b} y(t) d t=c\right\}=\frac{3(1-c)^{2}}{b-a} .
$$

Применяя лемму 2 к множеству $Y_{x}$ функций $y(t) \in W_{2,1}^{1}(-1,1)$ таких, что $y(x)=1$, по отдельности на каждом из отрезков $[-1, x]$ и $[x, 1]$ получаем равенство

$$
\min \left\{J[y]: y \in Y_{x}\right\}=3\left(\frac{(1-\widetilde{c})^{2}}{1+x}+\frac{(1-\widehat{c})^{2}}{1-x}\right)=: G(\widetilde{c}, \widehat{c}),
$$

где $\widetilde{c}, \widehat{c}$ - средние значения $y(t)$ на указанных отрезках, связанные соотношением

$$
(1+x) \widetilde{c}+(1-x) \widehat{c}=0,
$$

которое выражает условие ортогональности $y(t)$ константам на всем отрезке $[-1,1]$. 
Для нахождения минимума $G(\widetilde{c}, \widehat{c})$ при ограничении $(16)$ составляем функцию Лагранжа

$$
L(\widetilde{c}, \widehat{c}, \lambda):=3\left(\frac{(1-\widetilde{c})^{2}}{1+x}+\frac{(1-\widehat{c})^{2}}{1-x}\right)+\lambda((1+x) \widetilde{c}+(1-x) \widehat{c})
$$

и приравниваем нулю ее производные по $\lambda$ и $\widetilde{c}, \widehat{c}$, что приводит к линейной системе, состоящей из (16) и уравнений

$$
6(1-\widetilde{c})=\lambda(1+x)^{2}, \quad 6(1-\widehat{c})=\lambda(1-x)^{2} .
$$

Умножая первое равенство $(18)$ на $(1+x)$, а второе - на $(1-x)$ и складывая, получаем с учетом (16)

$$
12=\left((1+x)^{3}+(1-x)^{3}\right) \quad \text { влечет } \quad \lambda=\frac{6}{1+3 x^{2}} .
$$

Используя найденное значение $\lambda$, выводим из (18), что

$$
1-\widetilde{c}=\frac{(1+x)^{2}}{1+3 x^{2}}, \quad 1-\widehat{c}=\frac{(1-x)^{2}}{1+3 x^{2}},
$$

и подставляя эти величины в (15), приходим к соотношению (см. также (5))

$$
B_{0}^{-2}(x)=\min \{G(\widetilde{c}, \widehat{c}):(1+x) \widetilde{c}+(1-x) \widehat{c}=0\}=\frac{3\left((1+x)^{3}+(1-x)^{3}\right)}{\left(1+3 x^{2}\right)^{2}}=\frac{6}{1+3 x^{2}} .
$$

Если же $m=1$ и $x=0$ (случай (iii)), то из (20) следует, что $\widetilde{c}=\widehat{c}=0$, и экстремальная функция в (5) будет четной и поэтому автоматически ортогональной на $[-1,1]$ не только константам, но и первой степени $t$; следовательно, $B_{2}(0)=B_{1}(0)$.

ЗАмечание 1. Нетрудно вычислить (ср. (13)), что

$$
\widetilde{y}_{1}(0 ; t)=\frac{3}{2} t^{2}-3|t|+1
$$

ЗАмечание 2. Случаи, когда $m=2,0<|x|<1$, а, тем более, $m>2,|x|<1$, остаются пока не исследованными.

\section{СПИСОК ЦИТИРОВАННОЙ ЛИТЕРАТУРЫ}

[1] В. М. Тихомиров, Некоторые вопросы теории приближений, Изд-во Моск. ун-та, М., 1976. [2] Г. Бейтмен, А. Эрдейи, Высшие трансцендентные функиии. Гипергеометрическая функиия. Функиии Лежандра, Справочная математическая библиотека, Наука, М., 1973.

\section{Г. А. Калябин}

Поступило

Российский университет дружбы народов, г. Москва

02.03 .2011

E-mail: gennadiy.kalyabin@gmail.com 